\title{
A Membrane Fluidizing Factor in Sera from Duchenne Muscular Dystrophy Patients: Effect on Lymphocyte Membranes of Incubation in Patient and Control Lipoproteins
}

\author{
CHRISTOPH HÜBNER, ULRIKE WIEHLER, STEPHAN G. LINDNER, ULRIKE BEISIEGEL, \\ MATTHIAS CLAUSSEN, MICHAEL ALBANI, AND ALFRIED KOHLSCHÜTTER \\ Departments of Pediatrics [C.H., U.W., S.G.L., M.C., M.A., A.K.] and Internal Medicine [U.B.], University of \\ Hamburg, Hamburg, West Germany
}

\begin{abstract}
Sera from Duchenne muscular dystrophy (DMD) patients showed a membrane fluidizing effect on DMD and control lymphocytes. To look for the cause of this membrane fluidizing effect, human lymphocytes from healthy subjects were incubated in sera and in different serum fractions from DMD patients and healthy control subjects, and membrane fluidity was determined by steady state fluorescence polarization of the probe diphenyl hexatriene. DMD sera and total DMD lipoproteins showed a similar membrane fluidizing effect $(p<0.02)$ after incubation, whereas lipoprotein deficient serum did not show any effect on membrane fluidity. Control and DMD HDL showed a membrane fluidizing effect, the fluidizing effect of DMD HDL being slightly higher as compared to control HDL. Control LDL and DMD LDL showed a membrane rigidifying effect, the rigidifying effect of DMD LDL being significantly lower as compared to control LDL $(p<0.02)$. These data demonstrate that the membrane fluidizing effect of DMD serum is lipoprotein associated, LDL being the most important mediator of this effect. (Pediatr Res 25: 656-659, 1989)
\end{abstract}

\section{Abbreviations}

DMD, Duchenne muscular dystrophy DPH, 1,6-diphenyl-1,3,5-hexatriene LPDS, lipoprotein-deficient serum

The defective gene in DMD has been isolated (1), and the deficient gene product dystrophin has been characterized $(2,3)$. Dystrophin is expressed in all types of normal muscle cells $(4,5)$ whereas, except for central nervous system tissues, dystrophin expression in normal nonmuscle tissues correlates with smooth muscle myosin expression in these respective tissues (5). In DMD muscle biopsy specimens, dystrophin levels were less than $3 \%$ of normal levels (6). Dystrophin is localized at the cytoplasmic face of the plasma membrane of muscle fibers $(7-10)$ and shows some chemical and structural analogies with spectrin and with $\alpha$ actinin (3) probably indicating a cytoskeleton function (3). The localization of dystrophin in muscle cell plasma membranes (7) and the analogies of dystrophin to spectrin (3) may correlate to

Received August 29. 1988; accepted February 8, 1989.

Correspondence Dr. C. Hübner, Department of Pediatrics/Kinderklinik, University of Hamburg, Martinistr. 52, D-2000 Hamburg 20, West Germany.

Supported by Deutsche Forschungsgemeinschaft (Hu 408/1-4). A portion of this study is thesis work for U.W. other previous observations of "delta" lesions in DMD muscle membranes $(11,12)$. Erythrocyte spectrin is known to play an important role in membrane stability and fluidity $(13,14)$ and in maintenance of phospholipid asymmetry (15).

By contrast, indications for a defect in intracellular redox systems in DMD muscle cells are given by several studies. In avian muscular dystrophy, increased turnover of oxygen-derived free radicals is observed in the dystrophic muscle (16), and, in rat muscle cells, correlations between lipoxygenase activity and creatine kinase efflux are described (17). In DMD plasma lipid peroxidation products are elevated (18). Thus, it might be possible that dystrophin deficiency not only influences muscle membrane fluidity, but, as a secondary consequence lipid peroxidation in muscle cells and in serum fractions as well.

In a previous study, we showed that plasma membranes of DMD nonmuscle cells are not primarily affected, but that DMD sera contain a membrane-fluidizing factor (19). In the present study, we try to identify the membrane-fluidizing factor of DMD sera further, examining the influence of lipoproteins and of the different lipoprotein fractions on membrane fluidity. The results are discussed with respect to published alteration of DMD lipoproteins and to oxidation defects in DMD.

\section{MATERIALS AND METHODS}

Intact human lymphocytes from healthy subjects were isolated by Ficoll density gradient centrifugation according to Böyum (20). DPH fluorescence anisotropy was measured at $21^{\circ} \mathrm{C}$ in a Shimadzu spectrofluorophotometer RF 540. The preparation steps were done as described previously (19). Lymphocytes were incubated in the fluorescence marker DPH $\left(21^{\circ} \mathrm{C}\right)$ for $10 \mathrm{~min}$ and for $20 \mathrm{~min}$. Total fluorescence intensities $(21,22)$ were monitored to detect changes in fluorescence lifetime of DPH in lymphocytes after incubation in DMD versus control sera and serum fractions. In none of the measurements were such changes observed. Lipoproteins and LPDS were isolated by density gradient centrifugation using a modification of the method of Redgrave et al. (23). The density cuts were as follows: VLDL $(\mathrm{d}<$ $1.019 \mathrm{~g} / \mathrm{mL})$, LDL $(\mathrm{d}=1.045$ to $1.065 \mathrm{~g} / \mathrm{mL})$, HDL $(\mathrm{d}=1.095$ to $1.130 \mathrm{~g} / \mathrm{mL})$, and LPDS (d > $1.150 \mathrm{~g} / \mathrm{mL})$.

Incubations of lymphocytes in sera and in serum fractions were carried out at $4^{\circ} \mathrm{C}(19)$. For incubation experiments in total lipoproteins, DMD lipoproteins were added to control LPDS and vice versa. For incubation experiments in $\mathrm{HDL}, \mathrm{LDL}$, and VLDL, the respective DMD and control lipoprotein fractions were adjusted to an equal protein content with control LPDS. There were no differences in fluorescence anisotropy values between the incubation experiments performed with control 
versus DMD LPDS-adjusted lipoprotein fractions nor with autologous versus heterologous LPDS-adjusted lipoprotein fractions.

The age of the DMD patients was $10 \pm 4 \mathrm{y}$, the age of the controls was $20 \pm 9 \mathrm{y}$. The results correlated neither with the age of the control subjects nor with the age of the DMD patients nor with the general health conditions of the DMD patients.

Total cholesterol, free cholesterol, triglycerides, and phospholipids in sera and serum fractions were determined by standard enzymatic colorimetric methods (Diagnostica, Boehringer Mannheim GmbH, West Germany). Cholesteryl esters were estimated by comparing total cholesterol and free cholesterol contents. Extraction of lipids and determination of fatty acid methyl esters of lipoprotein fractions were done as described previously (24). Apoproteins were determined by single radial immunodiffusion (combi R.I.D., Immuno AG, Vienna, Austria).

Statistical analysis was carried out using the nonparametric Mann-Whitney test. The results are expressed as means \pm SD.

\section{RESULTS}

After incubation in DMD sera, DPH fluorescence anisotropy in lymphocyte plasma membranes was decreased as compared to the values after incubation in control sera (Fig. 1). After incubation in DMD total lipoproteins, fluorescence anisotropy in lymphocytes was decreased as compared to the values after incubation in control lipoproteins (Fig. 1). These data indicate a membrane fluidizing effect of DMD sera and lipoproteins. After incubation in DMD and control LPDS, no effect on DPH fluorescence anisotropy in lymphocytes was observed, indicating no change in membrane fluidity (Fig. 1).

After incubation in control HDL, fluorescence anisotropy values decreased as compared to initial values $(p<0.02$ for DPH $10 \mathrm{~min}$ and for DPH $20 \mathrm{~min}$ anisotropy values), indicating a membrane fluidizing effect of HDL (Fig. 1). When comparing the incubation effects of DMD versus control HDL, anisotropy values of DMD HDL were slightly lower versus controls (Fig. 1).

After incubation in control LDL, fluorescence anisotropy values increased as compared to initial values $(p<0.0002)$ indicating a membrane rigidifying effect of LDL (Fig. 1). When comparing the incubation effects of DMD versus control LDL, anisotropy values of DMD LDL were significantly lower versus controls (Fig. 1).

After incubation in control VLDL, fluorescence anisotropy values decreased as compared with initial values $(p<0.02)$,

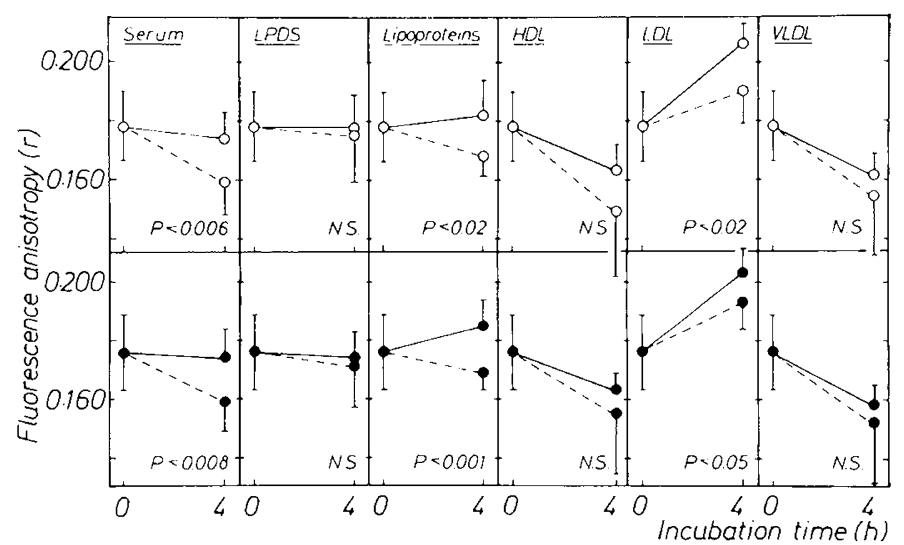

Fig. 1. Fluorescence anisotropy $\left(21^{\circ} \mathrm{C}\right)$ of DPH 10 min (marker in-

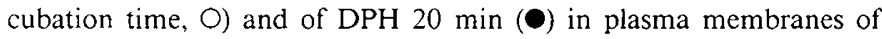
intact human lymphocytes from healthy subjects after incubation in control (—) vs. DMD (—- -) sera (controls $n=9$, DMD $n=10$ ), lipoprotein deficient sera (LPDS; controls $n=7$, DMD $n=9$ ), lipoproteins (controls $n=9$, DMD $n=10$ ), and lipoprotein fractions (controls $n=7$, DMD $n=9$ ), respectively; $p$ values for DMD $v s$. control data after a $4 \mathrm{~h}$ of incubation. indicating a membrane fluidizing effect of VLDL (Fig. 1). The incubation effects of DMD versus control VLDL on fluorescence anisotropy showed nearly no difference (Fig. 1).

Contents of cholesterol, triglycerides, phospholipids, and proteins in DMD lipoprotein fractions were not different from controls (Table 1). Fatty acid composition of DMD HDL was normal. In DMD, content of LDL C18:1 was increased, and in DMD VLDL, the contents of several fatty acids were different as compared to control VLDL (Table 2). In none of the DMD lipoprotein fractions were the wt ratios of saturated to cis-

Table 1. Composition of lipoprotein fractions used for the lymphocyte incubation experiments $(\mathrm{mg} / \mathrm{mL}$ serum)*

\begin{tabular}{lcc} 
& DMD-HDL & Control-HDL \\
Free cholesterol & $0.10 \pm 0.04(6 \%)$ & $0.10 \pm 0.04(4 \%)$ \\
Cholesteryl esters & $0.35 \pm 0.06(19 \%)$ & $0.52 \pm 0.09(20 \%)$ \\
Triglycerides $\dagger$ & $0.11(6 \%)$ & $0.07 \pm 0.03(3 \%)$ \\
Phospholipids & $0.37 \pm 0.11(20 \%)$ & $0.56 \pm 0.18(22 \%)$ \\
Apoprotein A-I & $0.7 \pm 0.2(38 \%)$ & $1.0 \pm 0.4(39 \%)$ \\
Apoprotein A-II & $0.2 \pm 0.1(11 \%)$ & $0.3 \pm 0.1(12 \%)$ \\
& DMD-LDL & Control-LDL \\
Free cholesterol & $0.14 \pm 0.05(6 \%)$ & $0.17 \pm 0.04(7 \%)$ \\
Cholesteryl esters & $1.03 \pm 0.26(47 \%)$ & $1.19 \pm 0.24(47 \%)$ \\
Triglycerides $\dagger$ & $0.13(6 \%)$ & $0.13 \pm 0.02(5 \%)$ \\
Phospholipids & $0.30 \pm 0.07(14 \%)$ & $0.33 \pm 0.11(13 \%)$ \\
Proteins & $0.59 \pm 0.12(27 \%)$ & $0.72 \pm 0.21(28 \%)$ \\
& DMD-VLDL & Control-VLDL \\
Free cholesterol & $0.04 \pm 0.02(3 \%)$ & $0.03 \pm 0.02(4 \%)$ \\
Cholesteryl esters & $0.26 \pm 0.17(17 \%)$ & $0.16 \pm 0.06(19 \%)$ \\
Triglycerides $\dagger$ & $1.03(67 \%)$ & $0.48 \pm 0.21(58 \%)$ \\
Phospholipids & $0.08 \pm 0.06(5 \%)$ & $0.08 \pm 0.05(10 \%)$ \\
Proteins & $0.12 \pm 0.06(8 \%)$ & $0.08 \pm 0.04(10 \%)$ \\
\hline
\end{tabular}

$* n=9$ for DMD and $n=7$ for control samples except for determinations of $\dagger$ triglycerides ( $n=2$ for DMD and $n=3$ for controls).

Table 2. Relative contents of fatty acids of lipoprotein fractions used for the lymphocyte incubation experiments*

\begin{tabular}{llccc}
\hline Subject & Fatty acids & $\begin{array}{c}\text { Wt } \% \\
\text { (HDL) }\end{array}$ & $\begin{array}{c}\text { Wt } \% \\
\text { (LDL) }\end{array}$ & $\begin{array}{c}\text { Wt } \% \\
\text { (VLDL) }\end{array}$ \\
\hline DMD & $16: 0$ & $23.8 \pm 4.0$ & $18.9 \pm 3.3$ & $27.5 \pm 2.1 \dagger$ \\
Controls & & $19.9 \pm 3.0$ & $24.1 \pm 5.3$ & $19.7 \pm 5.7$ \\
DMD & $18: 0$ & $11.6 \pm 1.8$ & $8.6 \pm 1.8$ & $9.3 \pm 1.9$ \\
Controls & & $10.9 \pm 1.7$ & $11.5 \pm 2.5$ & $9.1 \pm 1.4$ \\
DMD & $18: 1 \ddagger$ & $17.3 \pm 3.1$ & $19.7 \pm 1.2 \dagger$ & $29.7 \pm 3.3 \S$ \\
Controls & & $14.8 \pm 1.6$ & $15.1 \pm 2.9$ & $20.7 \pm 7.9$ \\
DMD & $18: 2 \|$ & $18.1 \pm 5.0$ & $21.7 \pm 7.2$ & $9.8 \pm 3.7 \S$ \\
Controls & & $21.9 \pm 6.0$ & $15.3 \pm 5.1$ & $14.8 \pm 2.6$ \\
DMD & $20: 3(\mathrm{n}-6) \uparrow$ & $1.0 \pm 0.5$ & $0.7 \pm 0.2$ & $0.4 \pm 0.3$ \\
Controls & & $1.1 \pm 0.5$ & $0.8 \pm 0.4$ & $0.7 \pm 0.2$ \\
DMD & $20: 4(\mathrm{n}-6)^{* *}$ & $3.6 \pm 1.8$ & $1.9 \pm 1.3$ & $0.9 \pm 0.6 \dagger$ \\
Controls & & $3.4 \pm 1.4$ & $1.7 \pm 0.6$ & $1.7 \pm 0.5$ \\
DMD & $22: 0$ & $0.3 \pm 0.1$ & $0.6 \pm 0.3$ & $0.3 \pm 0.2$ \\
Controls & & $0.5 \pm 0.3$ & $1.0 \pm 0.9$ & $0.6 \pm 0.6$ \\
DMD & $22: 6(\mathrm{n}-3) \dagger \dagger$ & $0.9 \pm 0.5$ & $0.3 \pm 0.2$ & $0.3 \pm 0.2$ \\
Controls & & $0.9 \pm 0.5$ & $0.6 \pm 0.8$ & $0.6 \pm 0.8$ \\
DMD & $24: 0$ & $0.3 \pm 0.1$ & $0.5 \pm 0.4$ & $0.3 \pm 0.2$ \\
Controls & & $0.4 \pm 0.3$ & $0.6 \pm 0.4$ & $0.5 \pm 0.3$ \\
\hline
\end{tabular}

${ }^{*} n=7$ for DMD and for control HDL; $n=4$ for DMD and for control LDL; $n=7$ for DMD and $n=5$ for control VLDL.

$\dagger p<0.05$ vs. controls.

$\ddagger 18: 1($ cis-9).

$\S p<0.02$ vs. controls.

|| $18: 2$ (cis, cis $-9,12)$.

T $20: 3$ (all cis-8,11,14).

** 20:4 (all cis-5, 8,11,14).

$\dagger \uparrow 22: 6$ (all cis-4,7,10,13,16,19). 
unsaturated fatty acids different from controls (data not shown). Protein content of total DMD lipoproteins $(31.9 \pm 4.4 \mathrm{mg} / \mathrm{mL}$; $n=5$ ) was not different from control lipoproteins $(32.3 \pm 5.7$ $\mathrm{mg} / \mathrm{mL} ; n=7)$.

\section{DISCUSSION}

After incubation in DMD sera, lymphocytes from DMD patients and from healthy controls showed an increased membrane fluidity due to a membrane fluidizing factor in DMD sera (19). Our present data on DMD serum incubation (Fig. 1) are in accordance with our previous results (19). The present incubation experiments demonstrate that DMD lipoproteins (Fig. 1), but not DMD LPDS, contain the membrane fluidizing factor which we previously proposed (19).

When using the different lipoprotein fractions, we found that after incubation in HDL, fluorescence anisotropy in lymphocytes decreased (Fig. 1); i.e. membrane fluidity increased, probably because cholesterol efflux from the cell is mediated by HDL (25). Our finding of an HDL-mediated fluidizing effect on lymphocyte membranes is in accordance with the finding that HDL-cholesterol has a fluidizing effect on erythrocyte membranes (26). The fluidizing effect of DMD HDL was slightly higher than that of control HDL (Fig. 1).

After incubation in the cholesterol-rich LDL (Table 1), fluorescence anisotropy increased (Fig. 1); i.e. membrane fluidity decreased, probably because cholesterol influx into cells is mediated by LDL (25). Our finding of a LDL-mediated rigidifying effect on lymphocyte membranes corresponds to the finding of a LDL-mediated increase of cholesterol content in lymphocyte (27) plasma membranes. An increased cholesterol content corresponds to increased DPH fluorescence anisotropy; i.e. decreased membrane fluidity (24). The rigidifying effect of DMD LDL was significantly lower than that of control LDL (Fig. 1).

Our findings demonstrate that the membrane fluidizing effect of DMD sera is lipoprotein-associated (Fig. 1). It is mainly caused by a less rigidifying effect of DMD LDL as compared to control LDL, but probably also by a more fluidizing effect of DMD HDL as compared to control HDL.

Regarding the incubation temperature of $4^{\circ} \mathrm{C}$, our findings are not likely to be due to enzymatic reactions. A major difference of lipid (Table 1) or of the fatty acid composition (Table 2) of DMD lipoproteins is also not likely to be the cause of the membrane fluidizing effect. In DMD HDL, neither fatty acid composition nor the saturated to cis-unsaturated fatty acid ratio were different versus controls, and, though there was an elevated content of C18:1 in DMD LDL (Table 2), the saturated to cisunsaturated fatty acid ratio was normal. Thus, a hitherto undetected difference in lipid composition of DMD LDL and HDL or an abnormal apoprotein could be responsible for the fluidizing effect of DMD lipoprotein fractions. Arthur et al. (28) found an increased electrophoretic mobility of DMD LDL. Increased electrophoretic LDL mobility can be due to an elevated content of the cis-unsaturated fatty acid C18:1 (29), to a structural aberration in the protein moiety (28), or to an increased lipid oxidation (30). We assume that the finding of Arthur et al. (28) of an abnormal electrophoretic mobility of DMD LDL and our finding of a membrane fluidizing effect of DMD LDL have the same biochemical cause. An increased C18:1 content (Table 2), therefore, is not likely to be the cause of the abnormal electrophoretic mobility (28), for, if it were the cause of the membrane fluidizing effect of DMD LDL, one would expect a decrease of the saturated to cis-unsaturated fatty acid ratio (24). An abnormal apoprotein also is not likely to be the cause of the abnormal electrophoretic mobility (28) nor of the fluidizing effect, as all the genes of the human apolipoproteins so far cloned are located on several autosomes, but not on chromosome $X(31)$. Thus, increased lipid oxidation is most likely the cause of the abnormal electrophoretic mobility (28) and the fluidizing effect of DMD LDL. This hypothesis is in accordance with the findings of elevated products of lipid peroxidation in DMD sera (18).
Steinbrecher et al. (30) demonstrated that cultured endothelial cells modify LDL in a way that leads to an increase of its electrophoretic mobility. They also showed that this modification of LDL involved free radical-initiated LDL peroxidation (30). In analogy to the findings of Steinbrecher et al. (30), it might be possible that DMD muscle tissue modifies LDL in a similar way. According to Morel et al. (32), oxidized LDL might be an important link between oxidative free radical generation and tissue damage. Although our results of the fluidizing effect of DMD LDL on lymphocytes do not correlate with the clinical stage of the patients, tissue damage by oxidized LDL nevertheless might add to the progradient clinical course of DMD.

Although we so far cannot further identify the membranefluidizing component in DMD LDL or HDL, it seems worthwhile to study in detail lipids and lipid oxidation in LDL and HDL from DMD patients. The pathogenesis of the clinical progression of DMD might be related to an interaction between dystrophin deficiency and lipoproteins.

Acknowledgments. The authors thank Dr. R. Beckmann, Department of Pediatrics, University of Freiburg, West Germany; Dr. E. Boltshauser, Department of Pediatrics, University of Zurich, Switzerland; Dr. F. Hanefeld, Department of Pediatrics, University of Göttingen, West Germany; Dr. H. G. Lenard, Department of Pediatrics, University of Düsseldorf, West Germany; Dr. W. Mortier, Children's Hospital, Wuppertal, West Germany for supplying biological material from DMD patients. The excellent technical assistance of M. Knorre and I. Wernicke is gratefully acknowledged.

\section{REFERENCES}

1. Koenig M. Hoffmann EP, Bertelson CJ Monaco AP, Feener C Kunkel LM 1987 Complete cloning of the Duchenne muscular dystrophy (DMD) cDNA and preliminary genomic organization of the DMD gene in normal and affected individuals. Cell 50:509-517

2. Hoffman EP, Brown RH Jr, Kunkel LM 1987 Dystrophin: the protein product of the Duchenne muscular dystrophy locus. Cell 51:919-928

3. Koenig M, Monaco AP, Kunkel LM 1988 The complete sequence of dystrophin predicts a rod-shaped cytoskeletal protein. Cell 53:219-228

4. Chelly J, Kaplan J-C. Maire P, Gautron S, Kahn A 1988 Transcription of the dystrophin gene in human muscle and non-muscle tissues. Nature 333:858 860

5. Hoffman EP, Hudecki MS, Rosenberg PA, Pollina CM, Kunkel LM 1988 Cel and fiber-type distribution of dystrophin. Neuron 1:411-420

6. Hoffman EP. Fischbeck KH, Brown RH, Johnson M, Medori R, Loike JD Harris JB, Waterston R, Brooke M, Specht L. Kupsky W, Chamberlain J, Caskey T, Shapiro F, Kunkel LM 1988 Characterization of dystrophin in muscle-biopsy specimens from patients with Duchenne's or Becker's dystrophy. N Engl J Med 318:1363-1368

7. Bonilla E, Samitt CE. Miranda AF, Hays AP, Salviati G, DiMauro S, Kunkel LM, Hoffman EP, Rowland LP 1988 Duchenne muscular dystrophy: deficiency of dystrophin at the muscle cell surface. Cell 54:447-452

8. Watkins SC, Hoffman EP, Slayter HS, Kunkel LM 1988 Immunoelectron microscopic localization of dystrophin in myofibres. Nature 333:863-866

9. Zubrzycka-Gaarn EE. Bulman DE. Karpati G. Burghes AHM, Belfall B, Klamut HJ, Talbot J, Hodges RS, Ray PN, Worton RG 1988 The Duchenne muscular dystrophy gene product is localized in sarcolemma of human skeletal muscle. Nature 333:466-469

10. Knudson CM, Hoffman EP, Kahl SD. Kunkel LM, Campbell KP 1988 Evidence for the association of dystrophin with the transverse tubular system in skeletal muscle. J Biol Chem 263:8480-8484

11. Mokri B, Engel AG 1975 Duchenne dystrophy: electron microscopic findings pointing to a basic or early abnormality in the plasma membrane of the muscle fiber. Neurology (NY) 25:1111-1120

12. Wakayama Y, Bonilla E, Schotland DL 1983 Muscle plasma membrane abnormalities in infants with Duchenne muscular dystrophy. Neurology (NY) 33:1368-1370

13. Farmer BT II, Harmon TM, Butterfield DA 1985 ESR studies of the erythrocyte membrane skeletal protein network: influence of the state of aggregation of spectrin on the physical state of membrane proteins, bilayer lipids, and cell surface carbohydrates. Biochim Biophys Acta 821:420-430

14. Waugh RE, Agre P 1988 Reductions of erythrocyte membrane viscoelastic coefficients reflect spectrin deficiencies in hereditary spherocytosis. $\mathrm{J}$ Clin Invest $81: 133-141$

15. Franck PFH, Op den Kamp JAF, Lubin B, Berendsen W, Joosten P, Briët E van Deenen LLM, Roelofsen B 1985 Abnormal transbilayer mobility of phosphatidylcholine in hereditary pyropoikilocytosis reflects the increased heat sensitivity of the membrane skeleton. Biochim Biophys Acta 815:259267

16. Ohta K, Muzuno Y 1988 Pathogenesis of progressive muscular dystrophy: 
studies on free radical metabolism in an animal model. Acta Neurol Scand $77: 108-114$

17. Jackson MJ, Wagenmakers AJM, Edwards RHT 1987 Effect of inhibitors of arachidonic acid metabolism on efflux of intracellular enzymes from skeletal muscle following experimental damage. Biochem J 241:403-407

18. Hunter MIS, Mohamed JB 1986 Plasma antioxidants and lipid peroxidation products in Duchenne muscular dystrophy. Clin Chim Acta 155:123-131

19. Hübner C Kohlschütter A, Gärtner J 1987 Membrane fluidity of nonmuscle cells in Duchenne muscular dystrophy: effect on lymphocyte membranes of incubation in patient and control sera. Pediatr Res 22:488-492

20. Böyum A 1968 Isolation of mononuclear cells and granulocytes from human blood. Scand J Clin Lab Invest 21 (suppl 97):77-89

21. Shinitzky M, Inbar M 1974 Difference in microviscosity induced by different cholesterol levels in the surface membrane lipid layer of normal lymphocytes and malignant lymphoma cells. J Mol Biol 85:603-615

22. Brasitus TA, Yeh K-Y, Holt PR, Schachter D 1984 Lipid fluidity and composition of intestinal microvillus membranes isolated from rats of different ages. Biochim Biophys Acta 778:341-348

23. Redgrave TG, Roberts DCK, West CE 1975 Separation of plasma lipoproteins by density-gradient ultracentrifugation. Anal Biochem 65:42-49

24. Hübner C, Lindner SG, Stern M, Claussen M, Kohlschütter A 1988 Membrane fluidity and lipid composition of rat small intestinal brush-border mem- branes during postnatal maturation. Biochim Biophys Acta 939:145-150 25. Fielding CJ, Fielding PE 1982 Cholesterol transport between cells and body fluids. Med Clin North Am 66:363-373

26. Kamada T, Otsuji S 1983 Lower levels of erythrocyte membrane fluidity in diabetic patients: a spin label study. Diabetes 32:585-591

27. Hambitzer R, Melzner I, Haferkamp O 1987 Relationships between lymphocyte cholesterol homeostasis and LDL-cholesterol. Clin Biochem 20:97-104 28. Arthur H, de Niese M, Jeffrey PL, Austin L 1983 Plasma lipoproteins in Duchenne muscular dystrophy. Biochem Int 6:307-313

29. Hayashi $H$, Naito C, Ito H, Kawamura M, Miyazaki S, Kumai M 1987 Enhanced degradation of low density lipoprotein in human monocytederived macrophages associated with an increase in its free fatty acid content. Atherosclerosis 66:139-144

30. Steinbrecher UP, Parthasarathy S, Leake DS, Witztum JL, Steinberg D 1984 Modification of low density lipoprotein by endothelial cells involves lipid peroxidation and degradation of low density lipoprotein phospholipids. Proc Natl Acad Sci USA 81:3883-3887

31. McAlpine PJ, Van Cong N, Boucheix C, Pakstis AJ, Doute RC, Shows TB 1987 The 1987 catalog of mapped genes and report of the nomenclature committee. Cytogenet Cell Genet 46:29-101

32. Morel DW, Hessler JR, Chisolm GM 1983 Low density lipoprotein cytotoxicity induced by free radical peroxidation of lipid. J Lipid Res 24:1070-1076 